\title{
Application for Superconvergence of Finite Element Approximations for the Elliptic Problem by Global and Local $L^{2}$-Projection Methods
}

\author{
Rabeea H. Jari, Lin Mu \\ Department of Applied Science, UALR, Little Rock, USA \\ Email: rhjari@ualr.edu, lxmu@ualr.edu
}

Received February 12, 2012; revised May 21, 2012; accepted July 12, 2012

\begin{abstract}
Numerical experiments are given to verify the theoretical results for superconvergence of the elliptic problem by global and local $L^{2}$-Projection methods.
\end{abstract}

Keywords: Finite Element Methods; Superconvergence; $L^{2}$-Projection; Elliptic Problem

\section{Introduction}

The elliptic problem seeks $u$ in a certain functional space such that

$$
\begin{array}{r}
-\Delta u=f \text { in } \Omega \\
u=g \text { in } \partial \Omega
\end{array}
$$

where $\Delta$ denote the Laplacian operator.

Let $T_{h}$ be a finite element partition of the domain $\Omega$ with characteristic mesh size $h$. Let $V_{h} \subset H_{g}^{1}(\Omega)$ be any finite element space for $u$ associated with the partition $T_{h}$.

The $L^{2}$-Projection technique was introduced by Wang [1-3]. It projects the approximate solution to another finite element dimensional space associated with a coarse mesh.

Now, we start with defining a coarse mesh $T_{\tau}$ where $\tau \square h$ satisfying:

$$
\tau=h^{\alpha}
$$

with $\alpha \in(0,1)$. Define finite element space $V_{\tau} \subset H^{s-2}(\Omega)$. Let $Q_{\tau}$ to be the $L^{2}$-Projector onto the finite element space $V_{\tau}[1,4,5]$. The Projector $Q_{\tau}$ can be considered as a linear operator (projection) from $L^{2}(\Omega)$ onto the finite element space $V_{\tau}[6,7]$.

\section{Superconvergence by Global

$$
L^{2} \text {-Projection }
$$

The following theorems can be found in [1].

Theorem 2.1: Assume that $1 \leq s \leq k+1$ and the finite element space $V_{\tau} \subset H^{s-2}(\Omega)$. If the exact solution $u \in H^{k+1}(\Omega) \cap H^{r+1}(\Omega) \cap H_{g}^{1}(\Omega)$, then there exists a constant $C$ such that

$$
\begin{aligned}
& \left\|u-Q_{\tau} u_{h}\right\|+h^{\alpha}\left\|\nabla_{\tau}\left(u-Q_{\tau} u_{h}\right)\right\| \\
& \leq C h^{\alpha(r+1)}\|u\|+C h^{\sigma}\left\|\nabla\left(u-u_{h}\right)\right\|,
\end{aligned}
$$

where $\sigma=s-1+\alpha \min (0,2-s)$ and $u_{h}$ is the finite element approximation of (1) and (2).

Theorem 2.2: Suppose that $1 \leq s \leq k+1$. Let the surface fitting spaces $V_{\tau} \subset H^{s-2}(\Omega)$ and $u_{h}$ be the finite element approximation of (1) and (2). Then, the postprocessing of $u_{h}$ is estimated by

$$
\alpha=\frac{k+s-1}{r+1-\min (0,2-s)} .
$$

\section{Numerical Experiments for Global $L^{2}$-Projection}

In this section, we present several numerical experiments to verify the theoretical analysis in [1]. The triangulation $T_{h}$ is constructed by: 1) dividing the domain into an $n^{3} \times n^{3}$ rectangular mesh; 2) connecting the diagonal line with the positive slope. Denote $h=\frac{1}{n^{3}}$ as the mesh size.

The finite element space is defined by $V_{h}=\left\{v \in H_{g}^{1}(\Omega) ;\left.v\right|_{K} \in P_{1}(K) ; \forall K \in T_{h}, v=g\right.$ on $\left.\partial \Omega\right\}$.

We define $V_{\tau}$ as follows:

$$
V_{\tau}=\left\{v \in L^{2}(\Omega):\left.v\right|_{K} \in P_{2}(K) ; \forall K \in T_{\tau}\right\} .
$$

Example 3.1: Let the domain $\Omega=[0,1] \times[0,1]$ and the exact solution is assumed as 


$$
u=x(1-x) y(1-y) .
$$

Table 1 shows that after the post-processing method, all the errors are reduced. The exact solution in $L^{2}$-norm of $\left\|u-Q_{\tau} u_{h}\right\|$ has the similar convergence rate as $\left\|u-u_{h}\right\|$. There is no improvement for the $u$ in $L^{2}$-norm. However, the error in $H^{1}$-norm have higher convergence rate, which is shown as $O\left(h^{1.3}\right)$ for $\left\|\nabla_{\tau}\left(u-Q_{\tau} u_{h}\right)\right\|$.
The order of convergence rate is $O\left(h^{0.3}\right)$ better than $\left\|\nabla\left(u-u_{h}\right)\right\|$, see Figures 1(a) and (b).

Figures 2(a) and (b) give results for the finite element approximation of (1)-(2) before and after post-processing.

Example 3.2: Let the domain $\Omega=[0,1] \times[0,1]$ and the exact solution is assumed as

$$
u=\sin (\pi x) \cos (\pi y) .
$$

Table 1. Errors on uniform triangular meshes $T_{h}$ and $T_{\tau}$.

\begin{tabular}{ccccc}
\hline$h$ & $\left|u-u_{h}\right|_{1}$ & $\left\|u-u_{h}\right\|$ & $\left|u-Q u_{h}\right|_{1}$ & $\left\|u-Q u_{h}\right\|$ \\
\hline $2^{-3}$ & $0.6632 \mathrm{e}-2$ & $0.1287 \mathrm{e}-3$ & $0.1427 \mathrm{e}-2$ & $0.1227 \mathrm{e}-3$ \\
$3^{-3}$ & $0.2799 \mathrm{e}-2$ & $0.2295 \mathrm{e}-4$ & $0.4332 \mathrm{e}-3$ & $0.2185 \mathrm{e}-4$ \\
$4^{-3}$ & $0.1433 \mathrm{e}-2$ & $0.6017 \mathrm{e}-5$ & $0.1763 \mathrm{e}-3$ & $0.5730 \mathrm{e}-5$ \\
$5^{-3}$ & $0.8294 \mathrm{e}-3$ & $0.2015 \mathrm{e}-5$ & $0.8504 \mathrm{e}-4$ & $0.1919 \mathrm{e}-5$ \\
$6^{-3}$ & $0.5223 \mathrm{e}-3$ & $0.7992 \mathrm{e}-6$ & $0.4596 \mathrm{e}-4$ & $0.7610 \mathrm{e}-6$ \\
$O(h)$ & 0.9998 & 1.9993 & 1.3504 & 1.9996 \\
\hline
\end{tabular}

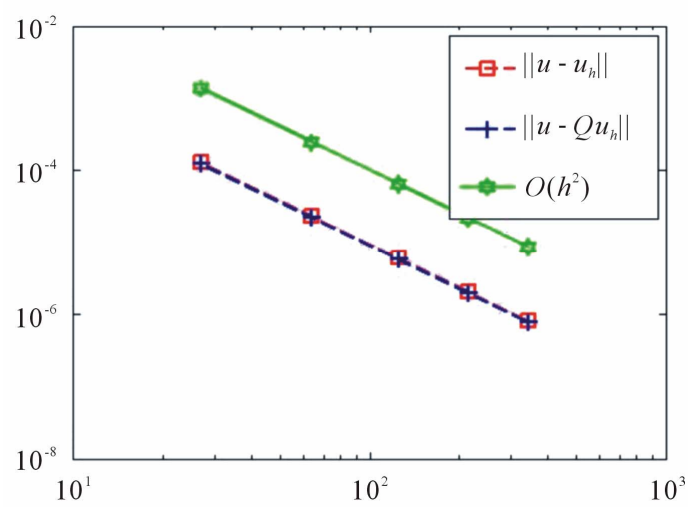

(a)

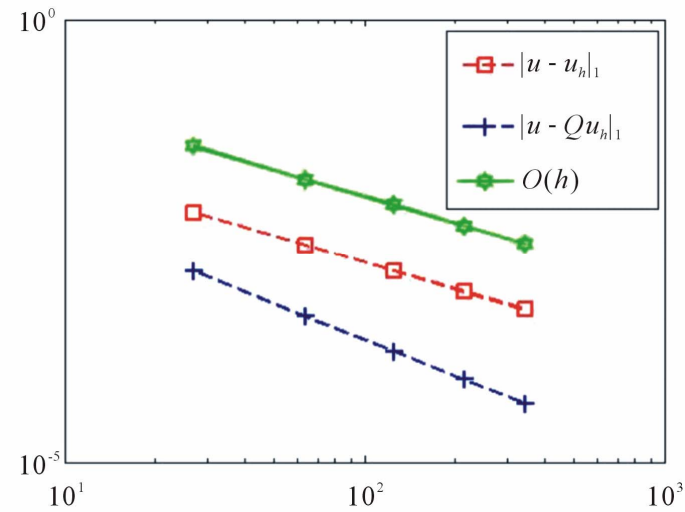

(b)

Figure 1. (a) Convergence rate of $L^{2}$-norm error; (b) Convergence rate of $H^{1}$-norm error.

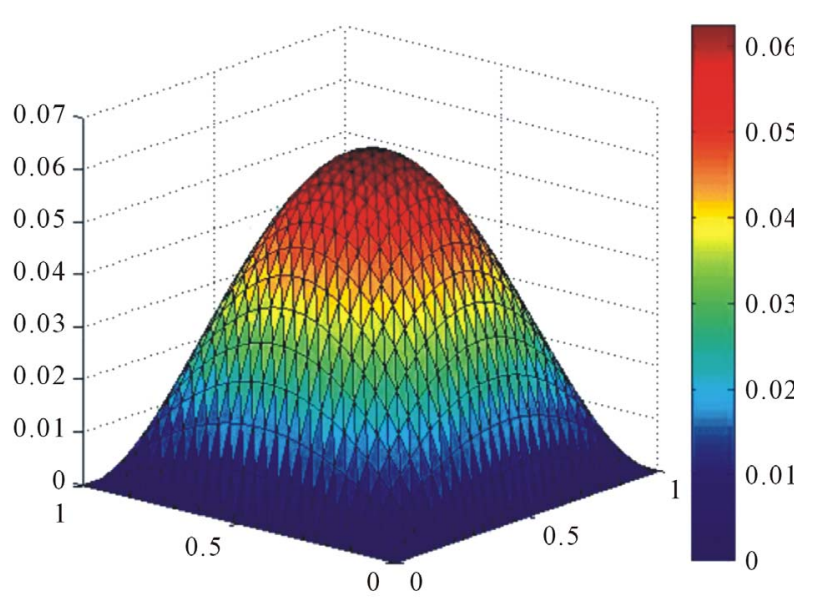

(a)

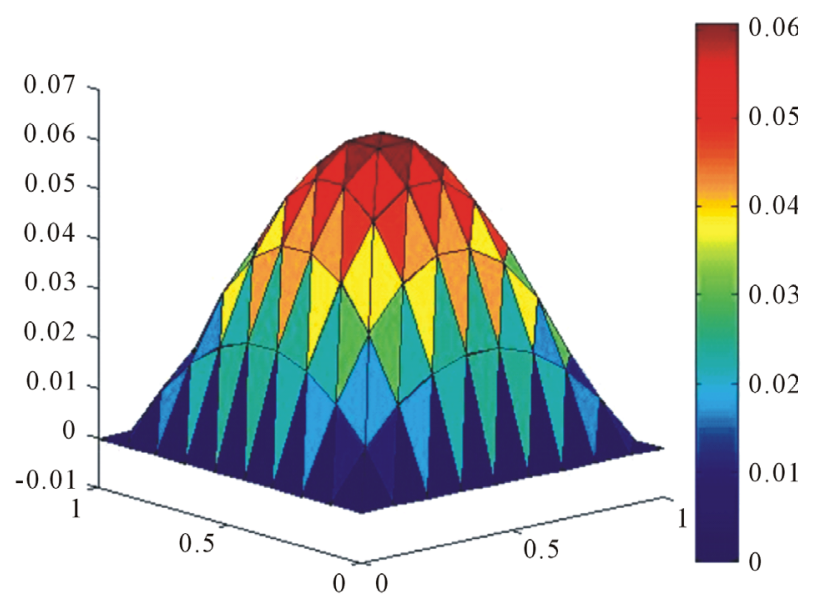

(b)

Figure 2. (a) Surface plot of approximation solution $u_{h}$; (b) Surface plot of approximation solution $Q_{\tau} u_{h}$. 
From the results shown in Table 2, it is clear that the exact solution $u$ in $H^{1}$-norm has the superconvergence, but there is no improvement in the $L^{2}$-norm, see Figures 3(a) and (b). The finite element solution given in Figures 4(a) and (b). This agrees well with the theory.

Example 3.3: Let the domain $\Omega=[0,1] \times[0,1]$ and the exact solution is assumed as

$$
u=\frac{\cos (\pi(x+y))}{2} \text {. }
$$

Table 3 gives the errors profile for Example 3. Notice that, the gradient estimate is of order $O\left(h^{1.3}\right)$, that is much better than the optimal order $O(h)$. Although, there is no improvement in the $L^{2}$-norm, see Figure 5.

Figure 6 shows that the approximation solutions $u_{h}$ and $Q_{\tau} u_{h}$.

Also, our numerical results and theoretical conclusions in Theorems (2.1) and (2.2) show highly consistent.

Table 2. Errors on uniform triangular meshes $T_{h}$ and $T_{\tau}$.

\begin{tabular}{ccccc}
\hline$h$ & $\left|u-u_{h}\right|_{1}$ & $\left\|u-u_{h}\right\|$ & $\left|u-Q u_{h}\right|_{1}$ & $\left\|u-Q u_{h}\right\|$ \\
\hline $2^{-3}$ & $0.9629 \mathrm{e}-1$ & $0.1598 \mathrm{e}-2$ & $0.2242 \mathrm{e}-1$ & $0.1498 \mathrm{e}-2$ \\
$3^{-3}$ & $0.4063 \mathrm{e}-1$ & $0.2850 \mathrm{e}-3$ & $0.6872 \mathrm{e}-2$ & $0.2669 \mathrm{e}-3$ \\
$4^{-3}$ & $0.2080 \mathrm{e}-1$ & $0.7475 \mathrm{e}-4$ & $0.2810 \mathrm{e}-2$ & $0.6998 \mathrm{e}-4$ \\
$5^{-3}$ & $0.1204 \mathrm{e}-1$ & $0.2503 \mathrm{e}-4$ & $0.1359 \mathrm{e}-2$ & $0.2343 \mathrm{e}-4$ \\
$6^{-3}$ & $0.7582 \mathrm{e}-2$ & $0.9929 \mathrm{e}-5$ & $0.7363 \mathrm{e}-3$ & $0.9294 \mathrm{e}-5$ \\
$O(h)$ & 0.9998 & 1.9991 & 1.3427 & 1.9995 \\
\hline
\end{tabular}

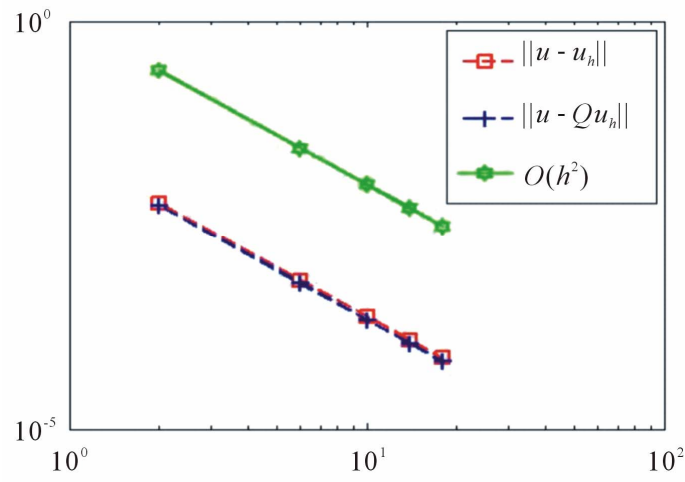

(a)

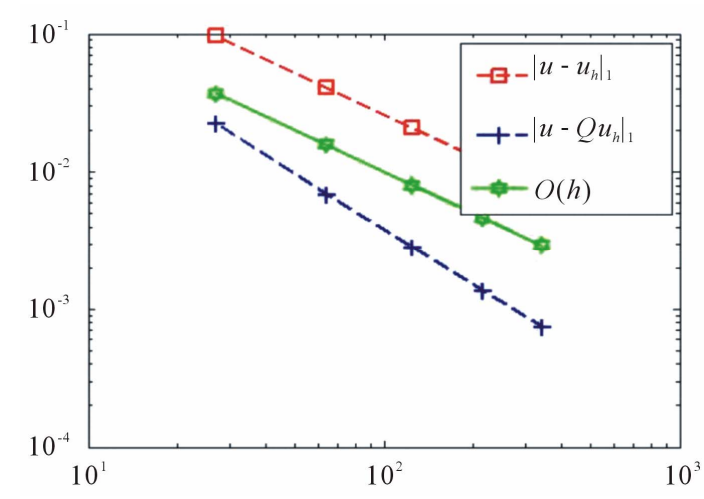

(b)

Figure 3. (a) Convergence rate of error $L^{2}$-norm error; (b) Convergence rate of $\boldsymbol{H}^{1}$-norm error.

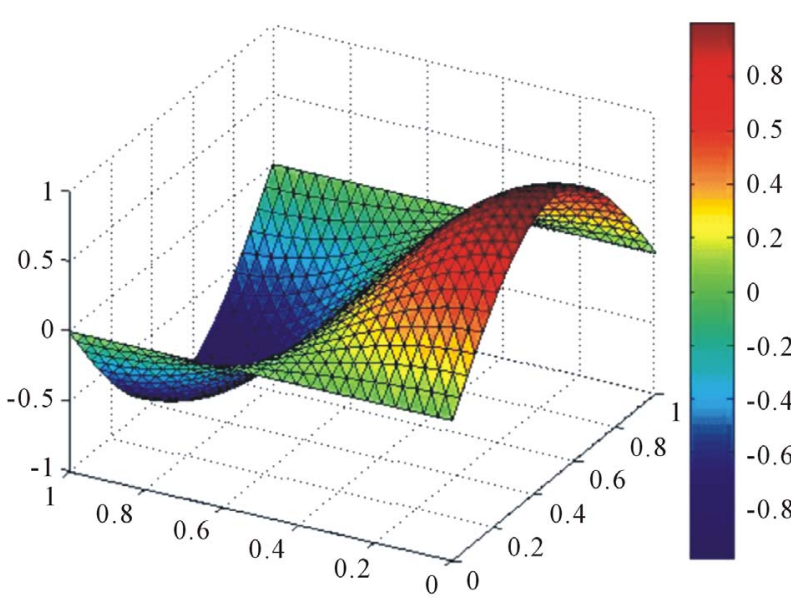

(a)

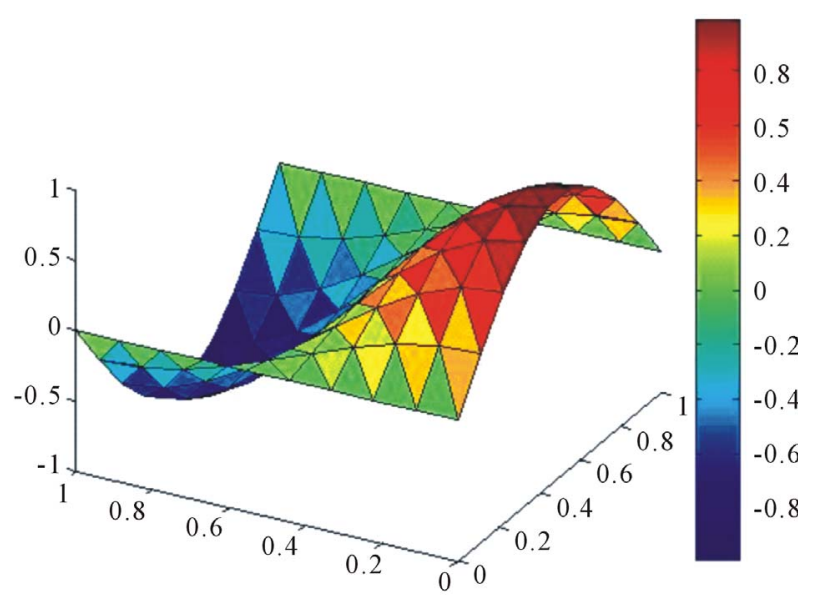

(b)

Figure 4. (a) Surface plot of solution $u_{h}$; (b) Surface plot of approximation solution $Q_{\tau} u_{h}$. 
Table 3. Errors on uniform triangular meshes $T_{h}$ and $T_{\tau}$.

\begin{tabular}{ccccc}
\hline$h$ & $\left|u-u_{h}\right|_{1}$ & $\left\|u-u_{h}\right\|$ & $\left|u-Q u_{h}\right|_{1}$ & $\left\|u-Q u_{h}\right\|$ \\
\hline $2^{-3}$ & $0.9135 \mathrm{e}-1$ & $0.1770 \mathrm{e}-2$ & $0.2150 \mathrm{e}-1$ & $0.1689 \mathrm{e}-2$ \\
$3^{-3}$ & $0.3855 \mathrm{e}-1$ & $0.3157 \mathrm{e}-3$ & $0.6579 \mathrm{e}-2$ & $0.3010 \mathrm{e}-3$ \\
$4^{-3}$ & $0.1973 \mathrm{e}-1$ & $0.8278 \mathrm{e}-4$ & $0.2692 \mathrm{e}-2$ & $0.7893 \mathrm{e}-4$ \\
$5^{-3}$ & $0.1142 \mathrm{e}-1$ & $0.2772 \mathrm{e}-4$ & $0.1303 \mathrm{e}-2$ & $0.2643 \mathrm{e}-4$ \\
$6^{-3}$ & $0.7193 \mathrm{e}-2$ & $0.1099 \mathrm{e}-4$ & $0.7062 \mathrm{e}-3$ & $0.1048 \mathrm{e}-4$ \\
$O(h)$ & 0.9999 & 1.9993 & 1.3424 & 1.9994 \\
\hline
\end{tabular}

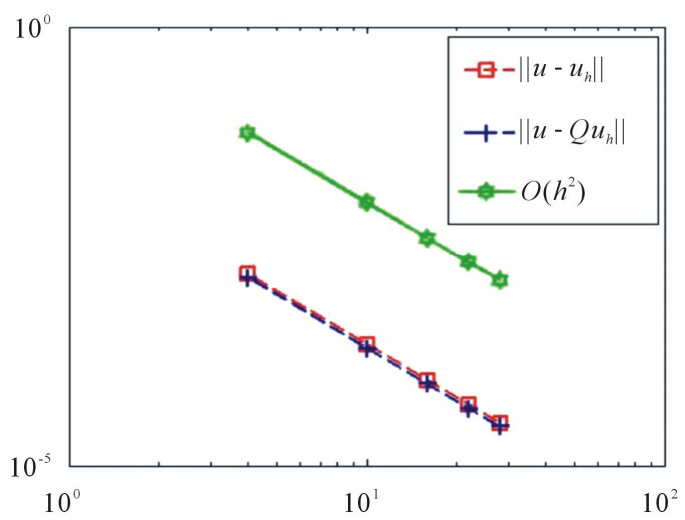

(a)

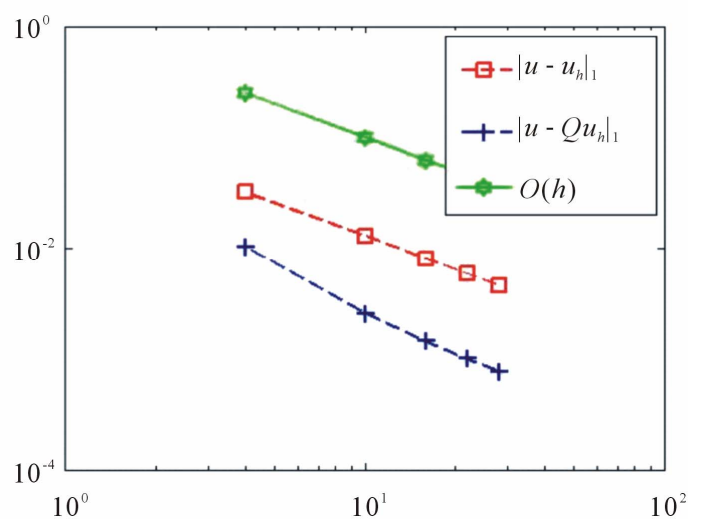

(b)

Figure 5. (a) Convergence rate of $L^{2}$-norm error; (b) Convergence rate of $H^{1}$-norm error.

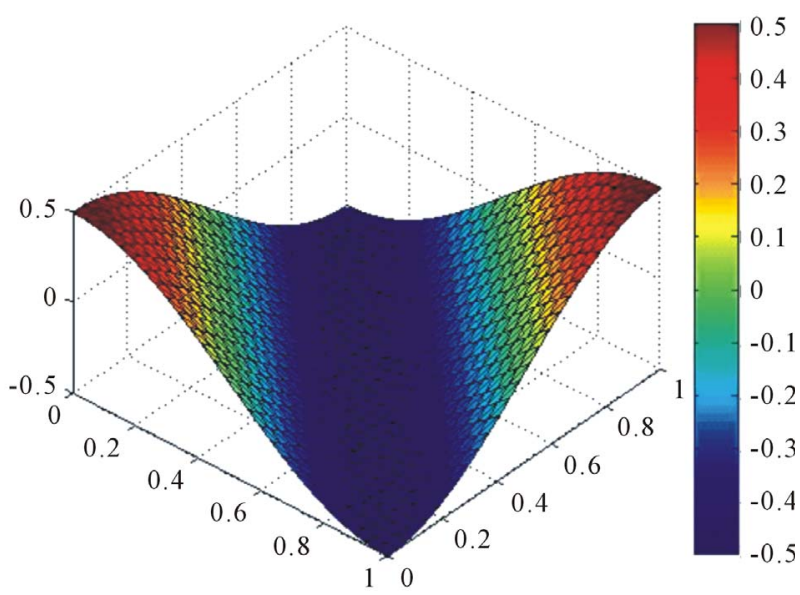

(a)

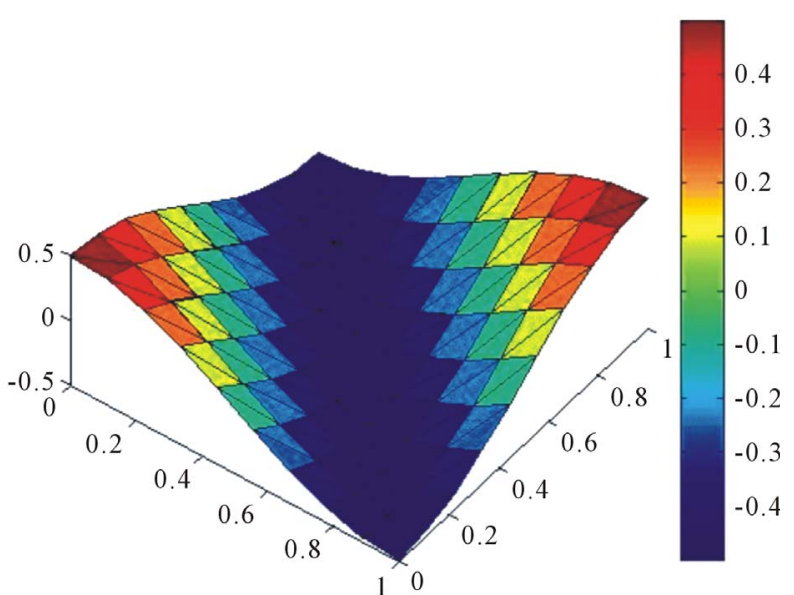

(b)

Figure 6. (a) Surface plot of approximation solution $u_{h}$; (b) Surface plot of approximation solution $Q_{\tau} u_{h}$.

\section{Superconvergence by Local $L^{2}$-Projection}

Notice that, the exact solution $u$ may be not smooth globally on $\Omega$ in practical computation, although the solution might be smooth enough locally for a good super convergence.

To this end, let $\Omega_{0}$ be a subdomain of $\Omega$ where the exact solution $u$ is sufficiently smooth. Let $\Omega_{1}$ be an- other subdomain of $\Omega$ such that $\Omega_{0} \subset \Omega_{1}$. Define finite element space $V_{\tau} \subset H^{s-2}\left(\Omega_{1}\right)$ The $L^{2}$-projection $Q_{\tau}$ from $L^{2}(\Omega)$ onto the finite element space $V_{\tau}$ is said to be local $L^{2}$-projection.

The following theorem can be found in [1].

Theorem 4.1: Assume that $1 \leq s \leq k+1$ and the finite element space $V_{\tau} \subset H^{s-2}\left(\Omega_{0}\right)$. If the exact solution $u \in H^{k+1}(\Omega) \cap H^{r+1}\left(\Omega_{0}\right) \cap H_{g}^{1}(\Omega)$, then there exists a 
constant $C$ such that

$$
\begin{aligned}
& \left\|u-Q_{\tau} u_{h}\right\|_{\Omega_{0}}+h^{\alpha}\left\|\nabla_{\tau}\left(u-Q_{\tau} u_{h}\right)\right\|_{\Omega_{0}} \\
& \leq C h^{\alpha(r+1)}\|u\|_{\Omega_{0}}+C h^{\alpha}\left\|\nabla\left(u-u_{h}\right)\right\|_{\Omega_{0}},
\end{aligned}
$$

where $u_{h}$ is the finite element approximation of (1)-(2).

Theorem 4.2: Suppose that $1 \leq s \leq k+1$. Let the surface fitting spaces $V_{\tau} \subset H^{s-2}\left(\Omega_{0}\right)$ and $u_{h}$ be the finite element approximation of (1)-(2). Then, the post-processing of $u_{h}$ is estimated by

$$
\alpha=\frac{k+s-1}{r+1-\min (0,2-s)} \text {. }
$$

\section{Numerical Experiments for Local $L^{2}$-Projection}

In this section, we present several numerical experiments to verify the theoretical analysis in [1]. The triangulation $T_{h}$ is constructed by: 1) dividing the domain into an $n^{3} \times n^{3}$ rectangular mesh; 2) connecting the diagonal line with the positive slope. Denote $h=\frac{1}{n^{3}}$ as the mesh size.

The finite element space is defined by

$V_{h}=\left\{v \in H_{g}^{1}(\Omega) ;\left.v\right|_{K} \in P_{1}(K) ; \forall K \in T_{h}, v=g\right.$ on $\left.\partial \Omega\right\}$.

We define $V_{\tau}$ as follows:

$$
V_{\tau}=\left\{v \in L^{2}(\Omega):\left.v\right|_{K} \in P_{2}(K) ; \forall K \in T_{\tau}\right\} .
$$

Example 5.1: Let the domain $\Omega=[0,1] \times[0,1]$ and $\Omega_{0}=[0,0.5] \times[0,0.5]$. The exact solution is assumed as

$$
u=\frac{1}{2-x-y} \text {. }
$$

It is clear that the exact solution $u$ is singular and $f$ blows down at the boundary of $\Omega=[0,1] \times[0,1]$, see

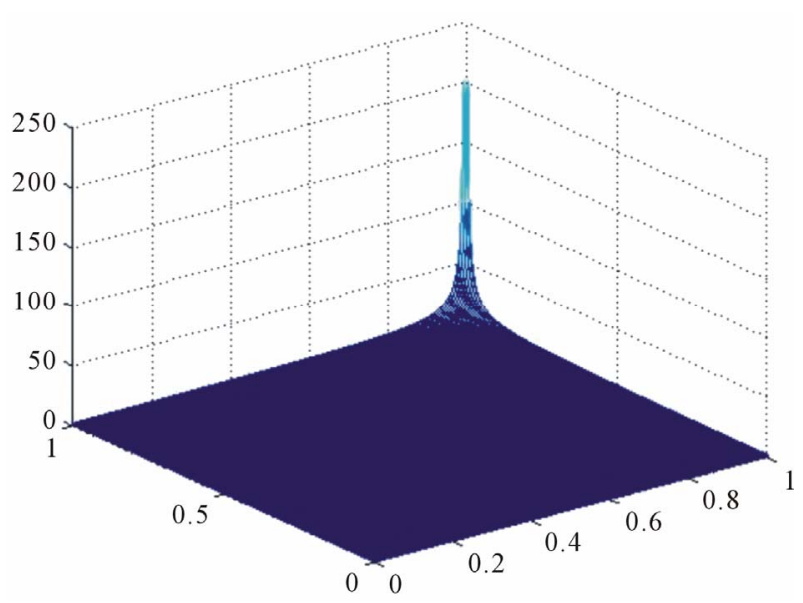

(a)
Figure 7, however, $u_{h}$ and $Q_{\tau} u_{h}$ are sufficiently smooth on $\Omega=[0,1] \times[0,1]$, see Figure 8 .

Table 4 shows that after the post-processing method, all the errors are reduced. The exact solution in $L^{2}$-norm of $\left\|u-Q_{\tau} u_{h}\right\|$ has the similar convergence rate as $\left\|u-u_{h}\right\|$ which is shown as $O\left(h^{2}\right)$. There is no improvement for the $u$ in $L^{2}$-norm. However, the error in $H^{1}$-norm have higher convergence rate, which is shown as $O\left(h^{1.3}\right)$ for $\left\|\nabla_{\tau}\left(u-Q_{\tau} u_{h}\right)\right\|$. The order of convergence rate is $O\left(h^{0.3}\right)$ better than $\left\|\nabla_{h}\left(u-u_{h}\right)\right\|$, see Figure 9.

Example 5.2: Let the domain $\Omega=[0,1] \times[0,1]$ and $\Omega_{0}=[0.5,1] \times[0.5,1]$. The exact solution is assumed as

$$
u=\sqrt{x^{2}+y^{2}}
$$

Obviously, the exact solution has singularity on the origin at the domain $\Omega=[0,1] \times[0,1]$, see Figure 10(a). On the same domain the function $f$ blows down at the boundary, see Figure 10(b). The approximation solutions $u$ and $Q_{\tau} u_{h}$ have been plot in the proper subdomain $\Omega_{0}=[0.5,1] \times[0.5,1]$, see Figure 11 .

From the results shown in Table 5, it is clear that the exact $u$ in $H^{1}$-norm has the superconvergence, but there is no improvement in the $L^{2}$-norm, see Figure 12. This agrees well with the theory.

Example 6: Let the domain $\Omega=[0,1] \times[0,1]$ and $\Omega_{0}=[0.5,1] \times[0.5,1]$. The exact solution is assumed as

$$
u=\frac{y}{\sqrt{x^{2}+y^{2}}} .
$$

From Figures 13(a) and (b), respectively observe that the exact solution has strongly singularity on the origin of the domain $\Omega=[0,1] \times[0,1]$ and the function $f$ blows up at the boundary, Figure 14 show how the approximation solution $u_{h}$ and $Q_{\tau} u_{h}$ look like at the proper subdomain $\Omega_{0}=[0.5,1] \times[0.5,1]$.

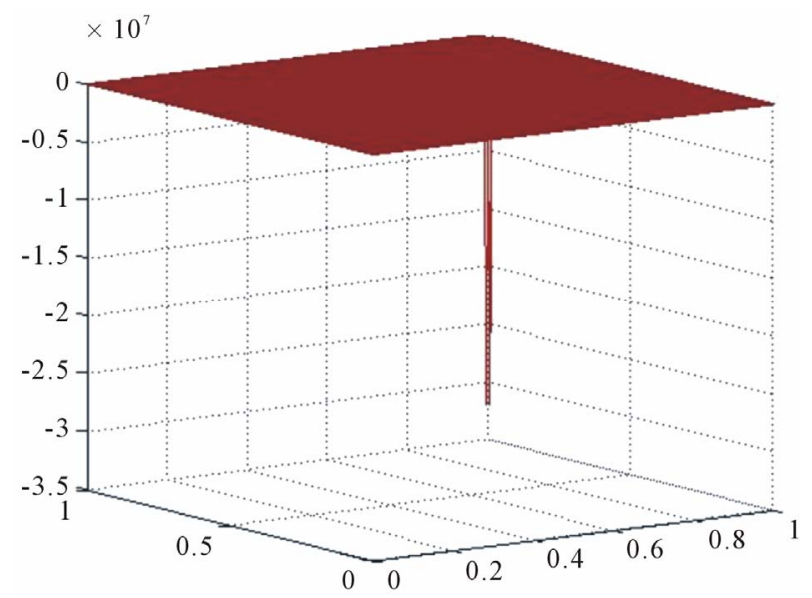

(b)

Figure 7. (a) The exact solution $u$ blows up; (b) $f$ blows down at the boundary. 


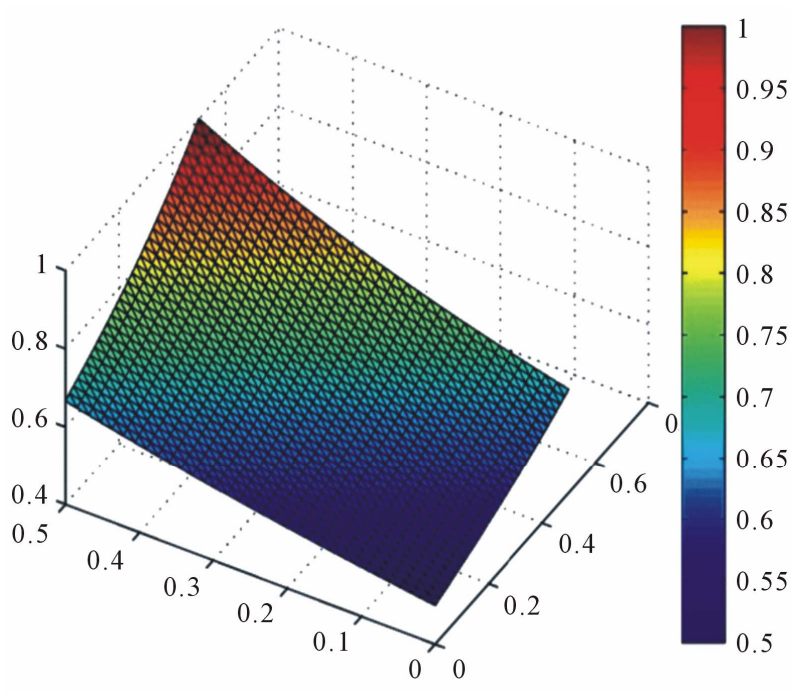

(a)

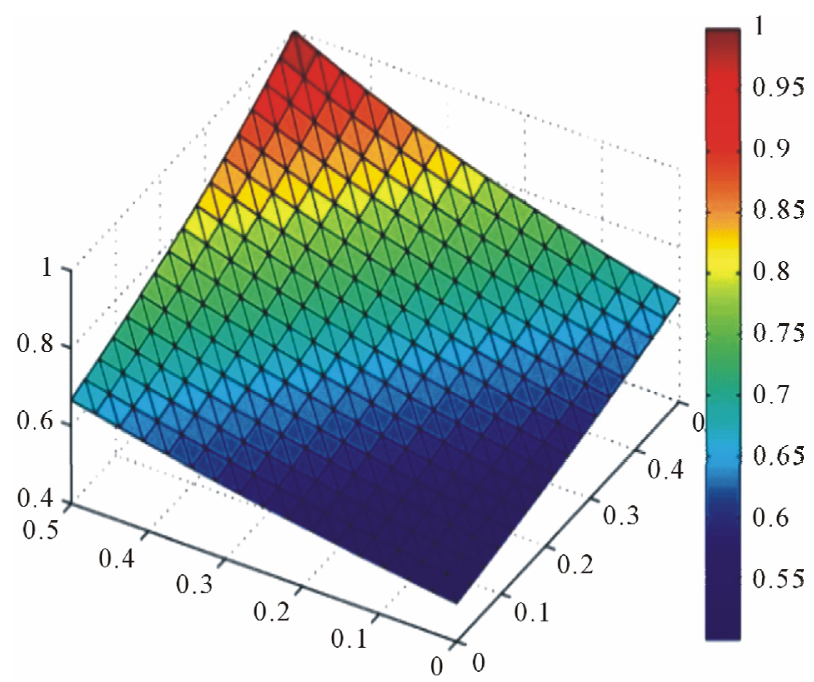

(b)

Figure 8. (a) Surface plot of approximation solution $u_{h}$; (b) Surface plot of approximation solution $Q_{\tau} u_{h}$.

Table 4. Errors on uniform triangular meshes $T_{h}$ and $T_{\tau}$.

\begin{tabular}{|c|c|c|c|c|}
\hline$h$ & $\left|u-u_{h}\right|_{1}$ & $\left\|u-u_{h}\right\|$ & $\left|u-Q u_{h}\right|_{1}$ & $\left\|u-Q u_{h}\right\|$ \\
\hline $2^{-3}$ & $0.3221 \mathrm{e}^{-1}$ & $0.1497 \mathrm{e}-2$ & $0.1026 \mathrm{e}^{-1}$ & $0.1363 \mathrm{e}-2$ \\
\hline $3^{-3}$ & $0.1291 \mathrm{e}^{-1}$ & $0.2384 \mathrm{e}^{-}-3$ & $0.2566 \mathrm{e}-2$ & $0.2169 \mathrm{e}-3$ \\
\hline $4^{-3}$ & $0.8072 \mathrm{e}-2$ & $0.9306 \mathrm{e}^{-4}$ & $0.1429 \mathrm{e}-2$ & $0.8466 \mathrm{e}^{-4}$ \\
\hline $5^{-3}$ & $0.5871 \mathrm{e}-2$ & $0.4921 \mathrm{e}^{-4}$ & $0.9977 \mathrm{e}-3$ & $0.4476 \mathrm{e}^{-4}$ \\
\hline $6^{-3}$ & $0.4613 \mathrm{e}-2$ & $0.3037 \mathrm{e}^{-4}$ & $0.7691 \mathrm{e}^{-3}$ & $0.2763 e^{-4}$ \\
\hline$O(h)$ & 0.9998 & 2.0030 & 1.3360 & 2.0035 \\
\hline
\end{tabular}

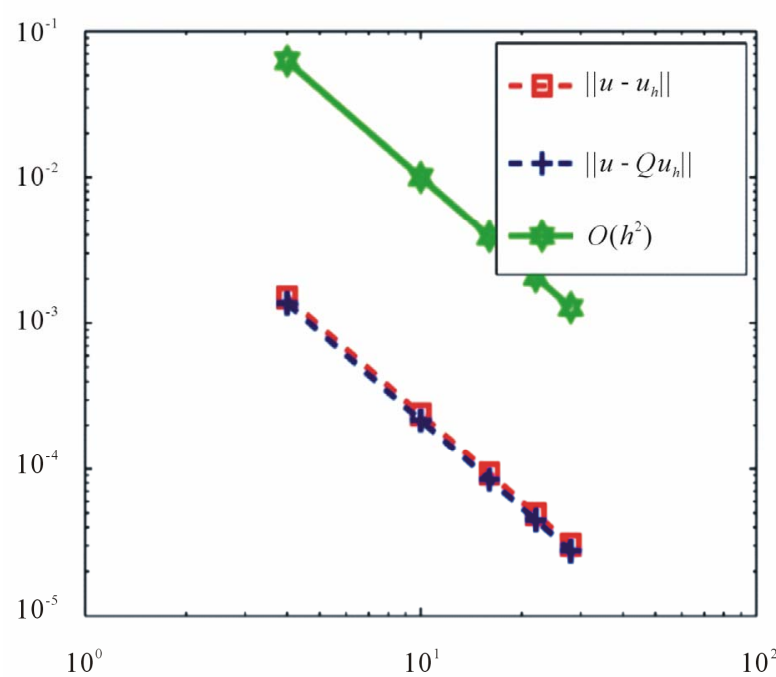

(a)

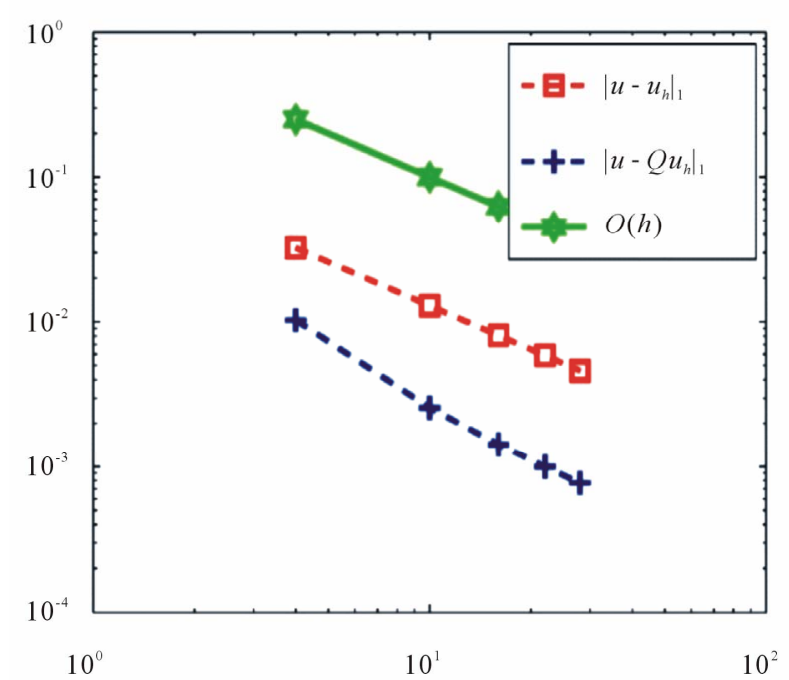

(b)

Figure 9. (a) Convergence rate of $L^{2}$-norm error; (b) Convergence rate of $H^{1}$-norm error. 


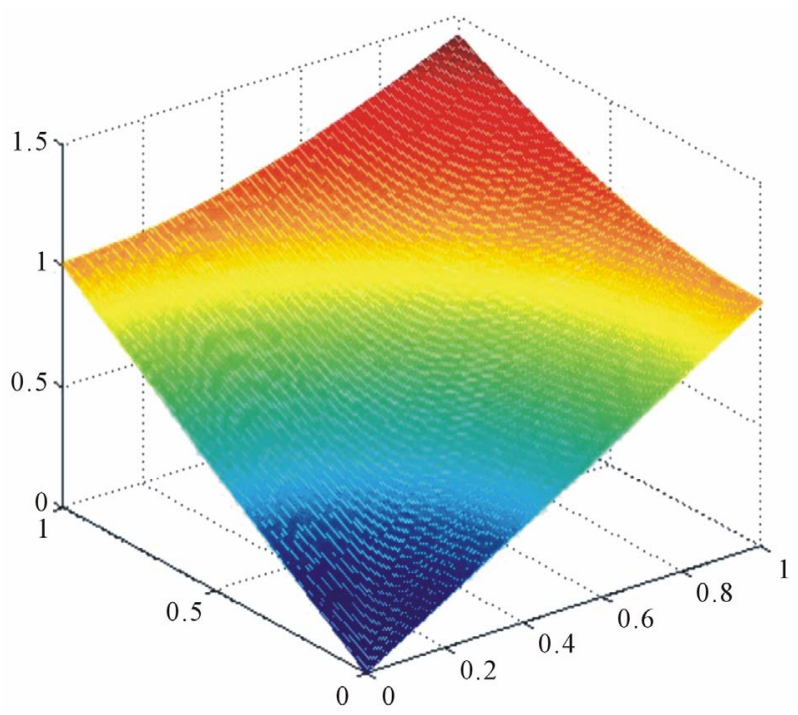

(a)

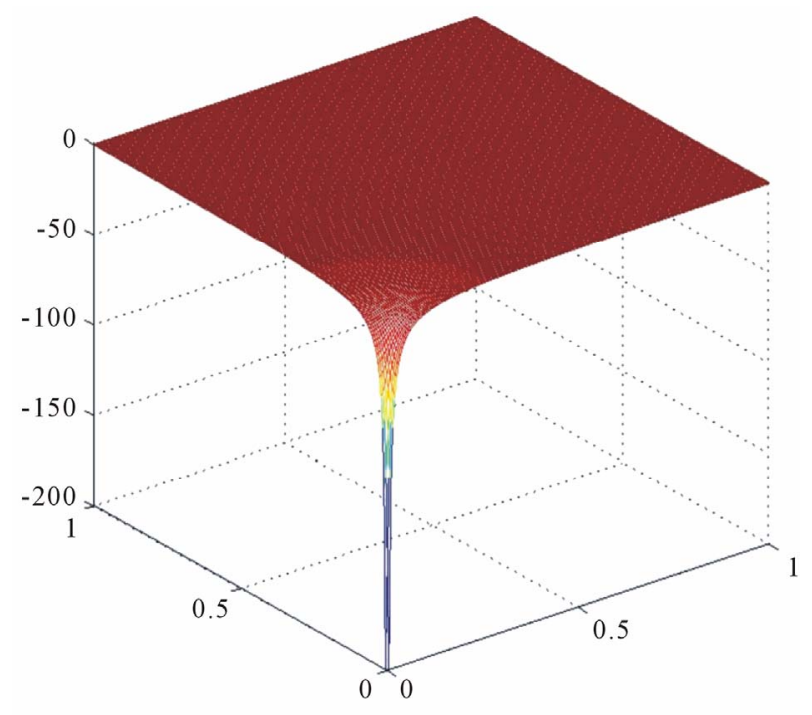

(b)

Figure 10. (a) Surface plot of exact solution $u$; (b) $f$ blows down at the boundary.

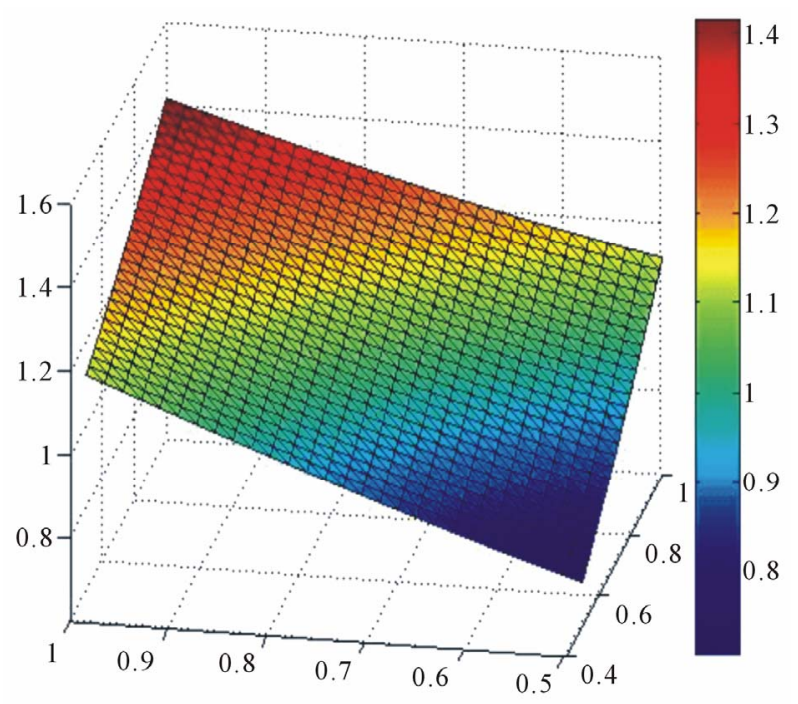

(a)

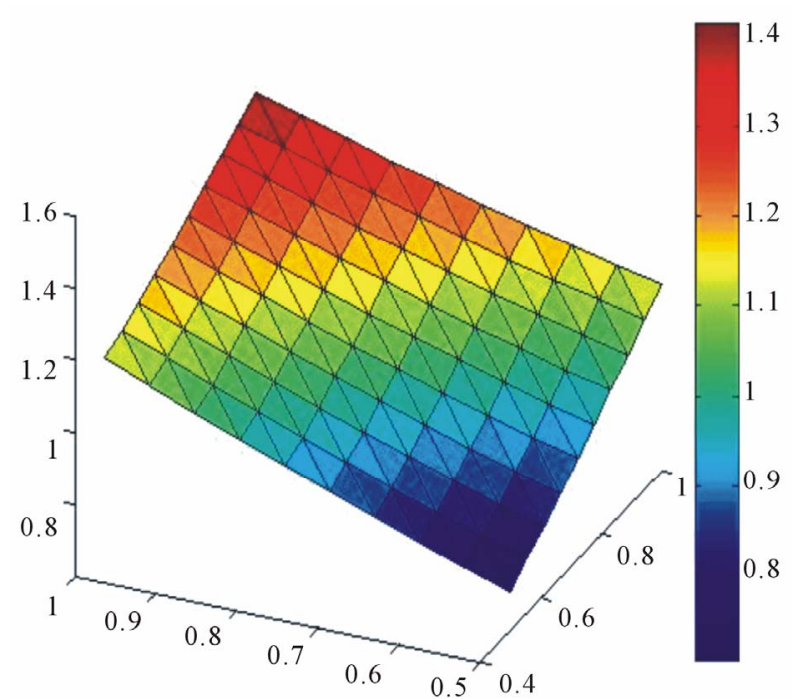

(b)

Figure 11. (a) Surface plot of approximation solution $u_{h}$; (b) Surface plot of approximation solution $Q_{\tau} u_{h}$.

Table 5. Errors on uniform triangular meshes $T_{h}$ and $T_{\tau}$.

\begin{tabular}{|c|c|c|c|c|}
\hline$h$ & $\left|u-u_{h}\right|_{1}$ & $\left\|u-u_{h}\right\|$ & $\left|u-Q u_{h}\right|_{1}$ & $\left\|u-Q u_{h}\right\|$ \\
\hline $2^{-3}$ & $0.1352 \mathrm{e}-1$ & $0.1400 \mathrm{e}-2$ & $0.6141 \mathrm{e}^{-2}$ & $0.1287 \mathrm{e}-2$ \\
\hline $3^{-3}$ & $0.6835 \mathrm{e}-2$ & $0.3596 \mathrm{e}^{-}-3$ & $0.2110 \mathrm{e}-2$ & $0.3314 \mathrm{e}^{-}-3$ \\
\hline $4^{-3}$ & $0.4566 \mathrm{e}-2$ & $0.1607 \mathrm{e}-3$ & $0.1215 \mathrm{e}-2$ & $0.1481 \mathrm{e}-3$ \\
\hline $5^{-3}$ & $0.3427 \mathrm{e}-2$ & $0.9058 \mathrm{e}-4$ & $0.8529 \mathrm{e}-3$ & $0.8352 \mathrm{e}-4$ \\
\hline $6^{-3}$ & $0.2743 \mathrm{e}-2$ & $0.5802 \mathrm{e}-4$ & $0.6590 e^{-3}$ & $0.5350 \mathrm{e}-4$ \\
\hline$O(h)$ & 0.9923 & 1.9806 & 1.3581 & 1.9792 \\
\hline
\end{tabular}




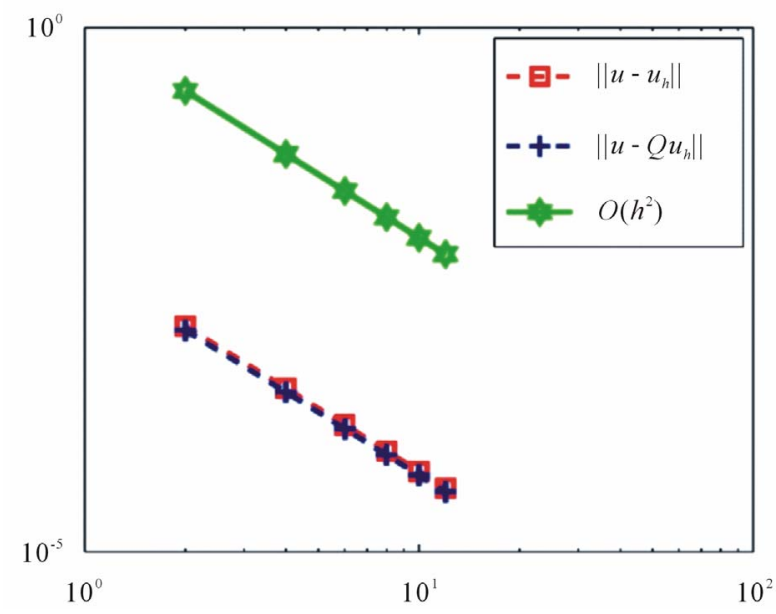

(a)

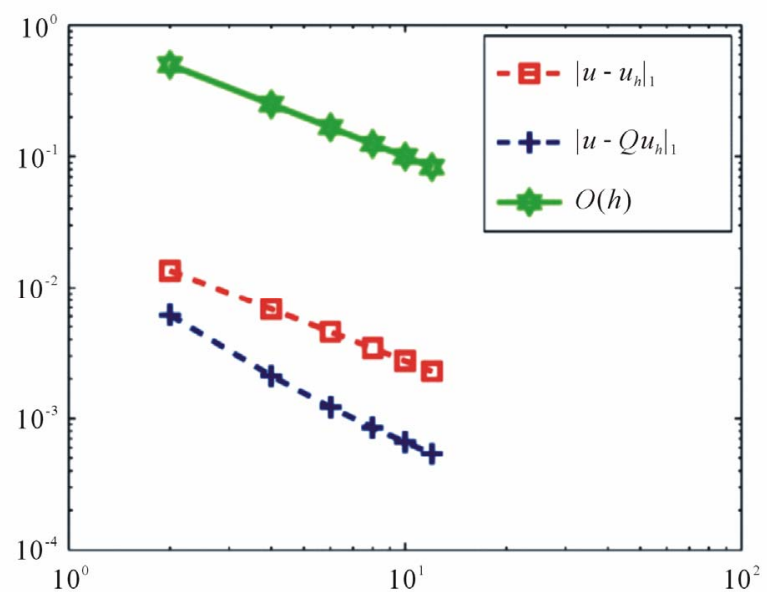

(b)

Figure 12. (a) Convergence rate of $L^{2}$-norm error; (b) Convergence rate of $H^{1}$-norm error.

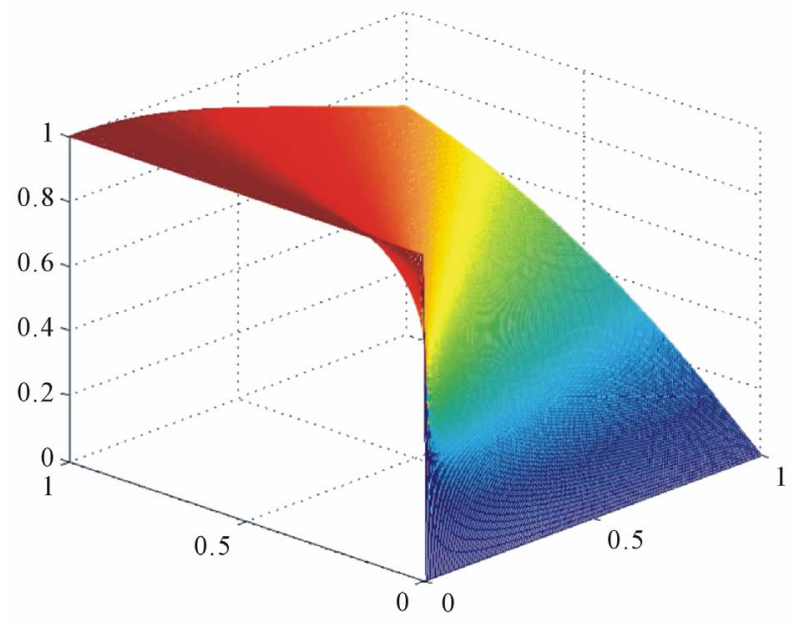

(a)

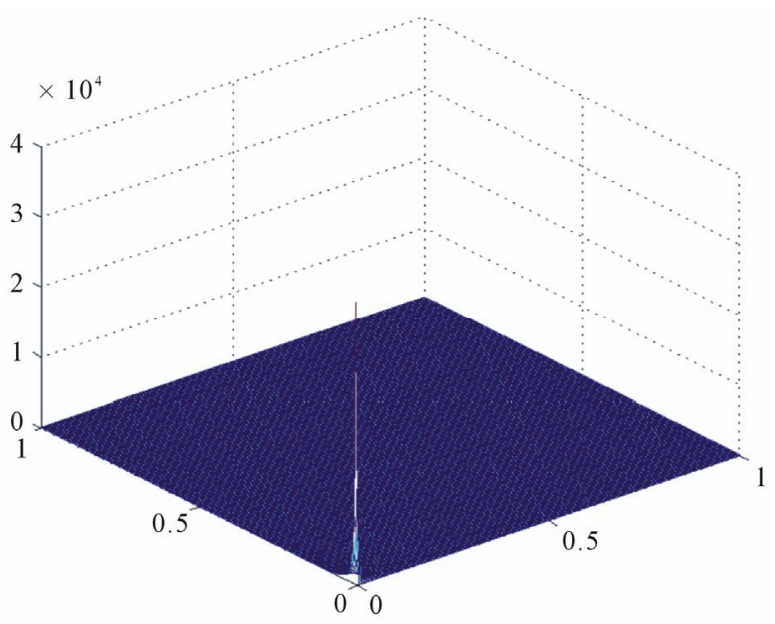

(b)

Figure 13. (a) Surface plot of exact solution $u$; (b) $f$ blows up at the boundary.

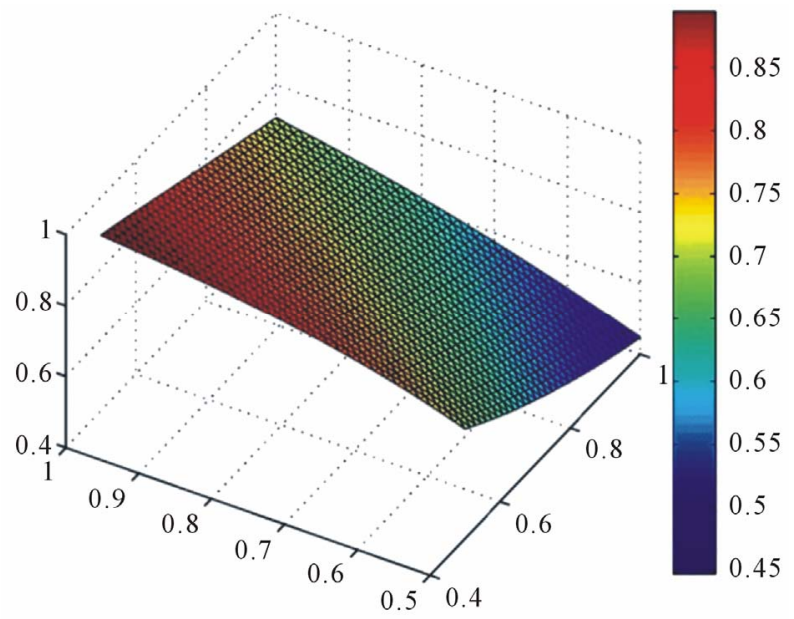

(a)

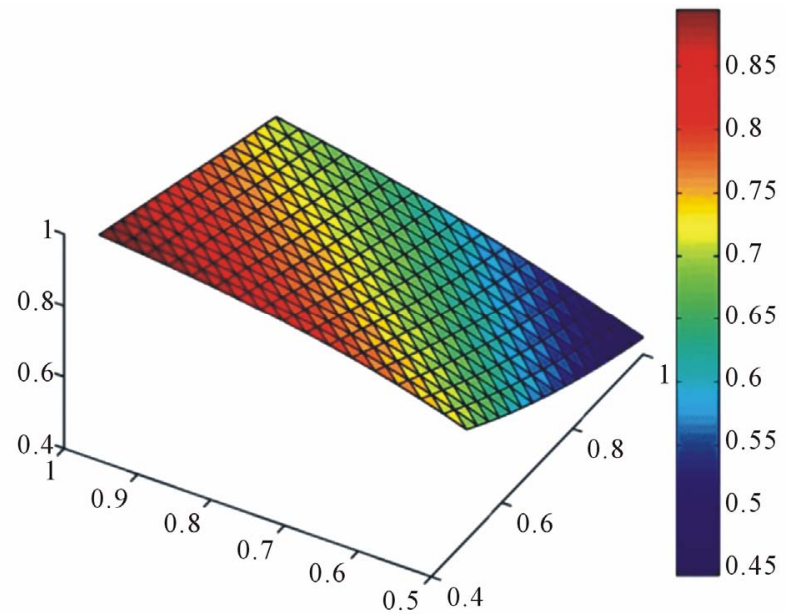

(b)

Figure 14. (a) Surface plot of approximation solution $u_{h}$; (b) Surface plot of approximation solution $Q_{\tau} u_{h}$. 
Table 6. Errors on uniform triangular meshes $T_{h}$ and $T_{\tau}$.

\begin{tabular}{ccccc}
\hline$h$ & $\left|u-u_{h}\right|_{1}$ & $\left\|u-u_{h}\right\|$ & $\left|u-Q u_{h}\right|_{1}$ & $\left\|u-Q u_{h}\right\|$ \\
\hline $2^{-3}$ & $0.1186 \mathrm{e}-1$ & $0.4006 \mathrm{e}-3$ & $0.4708 \mathrm{e}-2$ & $0.2779 \mathrm{e}-3$ \\
$3^{-3}$ & $0.5979 \mathrm{e}-2$ & $0.1009 \mathrm{e}-3$ & $0.1621 \mathrm{e}-2$ & $0.6959 \mathrm{e}-4$ \\
$4^{-3}$ & $0.3992 \mathrm{e}-2$ & $0.4490 \mathrm{e}-4$ & $0.9518 \mathrm{e}-3$ & $0.3094 \mathrm{e}-4$ \\
$5^{-3}$ & $0.2996 \mathrm{e}-2$ & $0.2527 \mathrm{e}-4$ & $0.6760 \mathrm{e}-3$ & $0.1740 \mathrm{e}-4$ \\
$6^{-3}$ & $0.2397 \mathrm{e}-2$ & $0.1617 \mathrm{e}-4$ & $0.5261 \mathrm{e}-3$ & $0.1113 \mathrm{e}-4$ \\
$O(h)$ & 0.9943 & 1.9949 & 1.3304 & 1.9989 \\
\hline
\end{tabular}

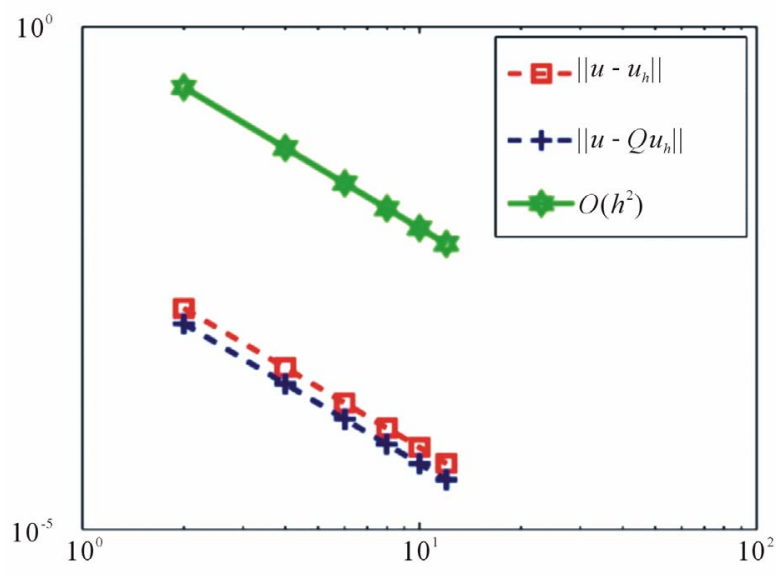

(a)

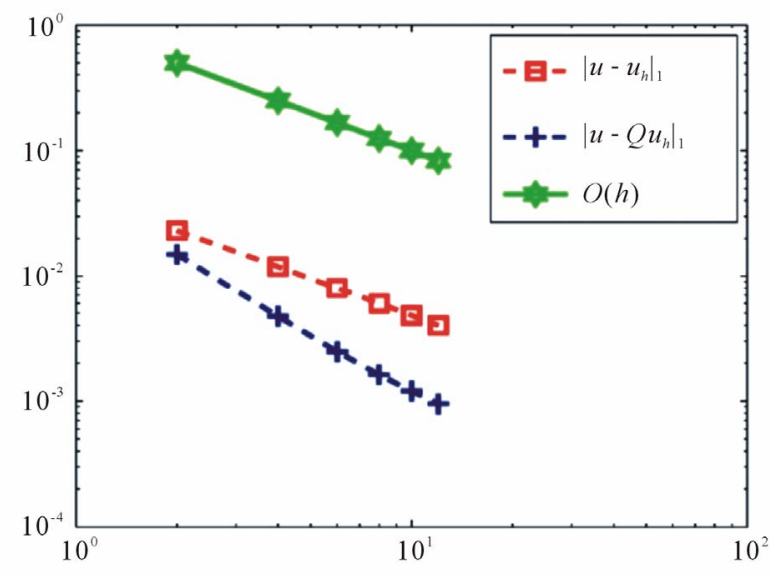

(b)

Figure 15. (a) Convergence rate of $L^{2}$-norm error; (b) Convergence rate of $H^{1}$-norm error.

Table 6 gives the errors profile for Example 6. Notice that, the gradient estimate is of order $O\left(h^{1.3}\right)$ that is much better than the optimal order $O(h)$. Although, there is no improvement in the $L^{2}$-norm, see Figure 15. Also, the numerical results and theoretical conclusions show highly consistent.

\section{REFERENCES}

[1] J. Wang, "A Superconvergence Analysis for Finite Element Solutions by the Least-Squares Surface Fitting on Irregular Meshes for Smooth Problems," Journal of Mathematical Study, Vol. 33, No. 3, 2000, pp. 229-243.

[2] R. E. Ewing, R. Lazarov and J. Wang, "Superconvergence of the Velocity along the Gauss Lines in Mixed Finite Element Methods," SIAM Journal on Numerical Analysis, Vol. 28, No. 4, 1991, pp. 1015-1029. doi:10.1137/0728054

[3] M. Zlamal, "Superconvergence and Reduced Integration in the Finite Element Method," Mathematics Computation, Vol. 32, No. 143, 1977, pp. 663-685. doi: $10.2307 / 2006479$

[4] L. B. Wahlbin, "Superconvergence in Galerkin Finite Element Methods," Lecture Notes in Mathematics, Springer, Berlin, 1995.

[5] A. H. Schatz, I. H. Sloan and L. B. Wahlbin, "Superconvergence in Finite Element Methods and Meshes that Are Symmetric with Respect to a Point," SIAM Journal on Numerical Analysis, Vol. 33, No. 2, 1996, pp. 505-521. doi:10.1137/0733027

[6] M. Krizaek and P. Neittaanmaki, "Superconvergence Phenomenon in the Finite Element Method Arising from Avaraging Gradients," Numerische Mathematik, Vol. 45, No. 1, 1984, pp. 105-116.

[7] J. Douglas and T. Dupont, "Superconvergence for Galerkin Methods for the Two-Point Boundary Problem via Local Projections," Numerical Mathematics, Vol. 21, No. 3, 1973, pp. 270-278. doi:10.1007/BF01436631 